\title{
EFFECT OF EARLY WATER DEFICITS ON NODULATION AND NITROGEN CONTENT OF INOCULATED SOYBEAN
}

\author{
J. A. Adjetey ${ }^{1 *}$ and B. N. G. Nxumalo ${ }^{2}$ \\ ${ }^{1}$ Department of Crop Science and Production, Botswana University of Agriculture and Natural Resources, \\ P/Bag 0027, Gaborone, Botswana \\ ${ }^{2}$ Discipline of Crop Science, University of KwaZulu-Natal, Private Bag X01, Scottsville, Pietermaritzburg, South Africa \\ *Corresponding author, E-mail: jaadjetey56@gmail.com
}

(Received: 23 March 2017, Accepted: 20 October 2017)

Keywords: Nitrogen fixation, Glycine max, water stress

\begin{abstract}
The study was conducted at the University of KwaZulu-Natal (29o 37' S, 30 23' E) from May to November 2012 under controlled environment conditions with three water regimes namely: well watered controls, mild water stress to $-1.4 \mathrm{MPa}$ and severe water stress to $-2.0 \mathrm{MPa}$, at the $\mathrm{V}_{4}(28 \mathrm{DAS})$ and $\mathrm{V}_{5}$ stages (35 DAS). Severe stress significantly reduced no. of nodule and root mass, leaf area, shoot dry mass and uptake, regardless of the stage. Mild stress on the other hand had little effect on this parameter as plants recovered on re-watering, to attain values similar to the control treatments. The $\mathrm{V}_{4}$ and $\mathrm{V}_{5}$ stages can recover from mild stress, but severe stress at both growth stages reduces nodulation and nitrogen uptake and this can lead to reduction in production potential of soybean.
\end{abstract}

\section{Introduction}

Nodulation and nitrogen fixation begin in the early vegetative stage of the crop but little work has focussed on this stage. It is reported that on the basis of production areas, the crop fixes up to $77 \%$ of all $\mathrm{N}$ by crop legumes (Herridge et al., 2008). Among the several factors affecting nitrogen fixation is water stress, which causes reduced yield and seed quality (Candogan and Yazgan, 2016). Such yield reductions operate through limitation of the symbiotic process resulting in decreased nodule formation and $\mathrm{N}$ fixation (Serraj et al., 1999). Legume productivity has been greatly depressed both by intermittent drought, which could occur at any time during the growing season, but mostly by terminal drought, which occurs when stored soil moisture is depleted resulting in crop senescence (Serraj et al., 1999). The response of crops to water deficits is dependent upon the severity and duration of exposure, as well as the physiological stage at which it occurs (Desclaux et al., 2000). As the soybean plant grows from the time of flowering to seed enlargement, it shows little ability to compensate for stressful conditions and hence yield reductions are more likely with any stress imposed in this period (Khadem et al., 1985). Vegetative development of soybean was reportedly less sensitive to drought stress than the reproductive stages (Atti et al., 2004). The aim of this study was examine the response of soybean to water deficits at the $V_{4}$ and $V_{5}$ stages which occur about 21 and 45 days after sowing depending on growing conditions (Castell, 2010). Although nodulation begins about 14 days after sowing i.e. the $V_{1}$ stage, there is very little nitrogen fixation at the stage. Nitrogen fixation increases greatly during the $V_{4}$ and $V_{5}$ stages where nodules are large and active (Staton, 2011). Since nodules are active at these stages, it is 
important to examine how water deficits during that time impact on overall nodulation potential and nitrogen content of the crop.

\section{Materials and Methods}

The study was conducted at the University of KwaZulu-Natal (29 $37^{\prime}$ S, 30 $33^{\circ}$ ' E) from May to November 2012. Soybean cultivar PAN 1652 which matures in 120 days was used. The soil was obtained from a site at the University Experimental Farm where no fertilizer had been applied for a few years prior to the study. A commercial preparation of Bradyrhizobium japonicum strain W74 was used as a soil inoculants. The plants were grown in $1 \mathrm{~L}$ pots each containing about $1 \mathrm{~kg}$ of soil. All pots were seeded and thinned to one plant per pot about one week after emergence. The plants were grown in a growth room under well watered conditions and water stress treatments commenced at the $V_{4}$ and $V_{5}$ stages i.e. 28 and 35 DAS, respectively. The day/night temperatures in the growth room were $28 / 20{ }^{\circ} \mathrm{C}$ respectively, the photoperiod was $14 \mathrm{~h}$ day length and relative humidity was $60 \%$.

The experiment was a factorial combination of two vegetative stages $\left(V_{4}\right.$ and $\left.V_{5}\right)$ and three water treatments in a completely randomised design with six replications. The water treatments comprised of a well-watered control with leaf water potential of $-0.3 \mathrm{MPa}$, a mild stress in which water was withheld until the leaf water potential dropped to $-1.4 \mathrm{MPa}$ and a severe stress in which water was withheld until the leaf water potential was $-2 \mathrm{MPa}$. The water potential measurements were taken in the afternoon on the last expanded leaf, using a pressure chamber (Marti ez et al., 2013). In each measurement, a young fully expanded leaf was excised for the plant, covered in a plastic sheet and inserted into the pressure chamber. The gas connected to the pressure chamber was then opened to create pressure in the chamber. A hand lens was used to observe the excised section of the exposed leaf petiole. The water potential was recorded when a water bubble was observed to be exuded from the leaf petiole. After every stress treatment the plants were re-watered and leaf water potential approximating - $0.3 \mathrm{MPa}$ was maintained till the final harvest.

Plant sampling started at the beginning of the reproductive stage $\left(\mathrm{R}_{1}\right.$ i.e. $\left.55 \mathrm{DAS}\right)$ when soybean had initiated flowering. The Leaf area was measured using a portable leaf area meter (LI-3000, LI-COR Biosciences) equipped with an LI-3050A belt conveyor. Number of nodes $r$ and shoot dry mass was determined by drying at $70{ }^{\circ} \mathrm{C}$ for two days. Plant tissue nitrogen concentration was determined by the Dumas combustion method using a Tru Spec CN -

elemental analyzer (LECO Corporation). Nitrogen uptake was then calculated as a product of above ground dry weight and tissue nitrogen concentration [i.e. Nitrogen uptake $(\mathrm{g})=$ Dry mass (g) $x \mathrm{~N}(\%) / 100]$. The soil moistened with water and carefully removed from pots and the soil was washed off leaving clean roots. The total nodule number, nodule mass and root mass were determined.

\section{Results and Discussion}

Nodule number, dry mass and root mass were significantly lowered $(p<0.01)$ by severe water stress to $-2.0 \mathrm{MPa}$, compared to the other treatments (Fig. 1). There was little difference in response to severe stress ( $p>0.05$ ) whether it was imposed at the V4 or V5 stages. 

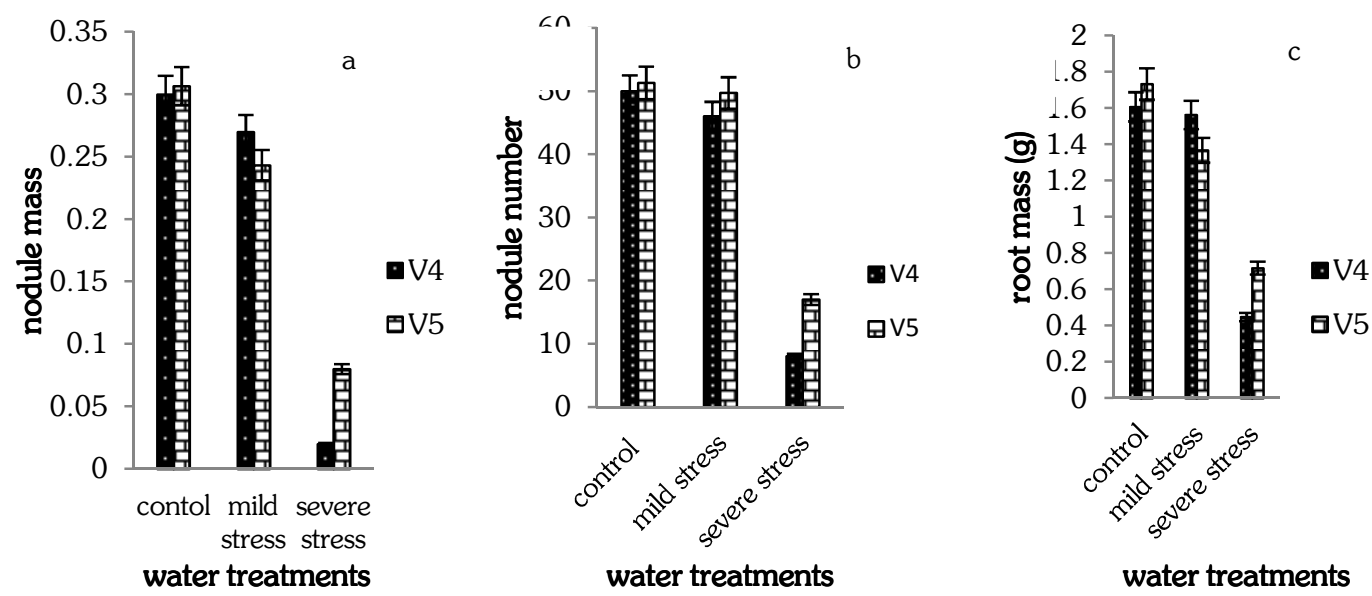

Fig. 1. Effect of water deficits on nodule mass (a) nodule number (b) and root mass (c) of soybean grown in controlled environment conditions.

Severe water stress significantly reduced $(p<0.01)$ number of nodes, shoot dry mass and leaf area (Figures 2 and 3). Plants which were stressed to $-1.4 \mathrm{MPa}$ were not different from the well watered controls but severe stress had a more detrimental effect. The effect was similar whether it was imposed at the $V_{4}$ or $V_{5}$ stage. There was no interaction between water stress treatments and the stage at which they were imposed.

There was no significant difference in shoot nitrogen concentration in respect of degree of stress or stage of application ( $p>0.05$ ), neither was there any interaction between the two factors. However, nitrogen uptake was significantly reduced $(p<0.001)$ by the severe water stress (Fig. 4). Plants stressed to -1.4 MPa was comparable with the well watered ones terms of in uptake of nitrogen.
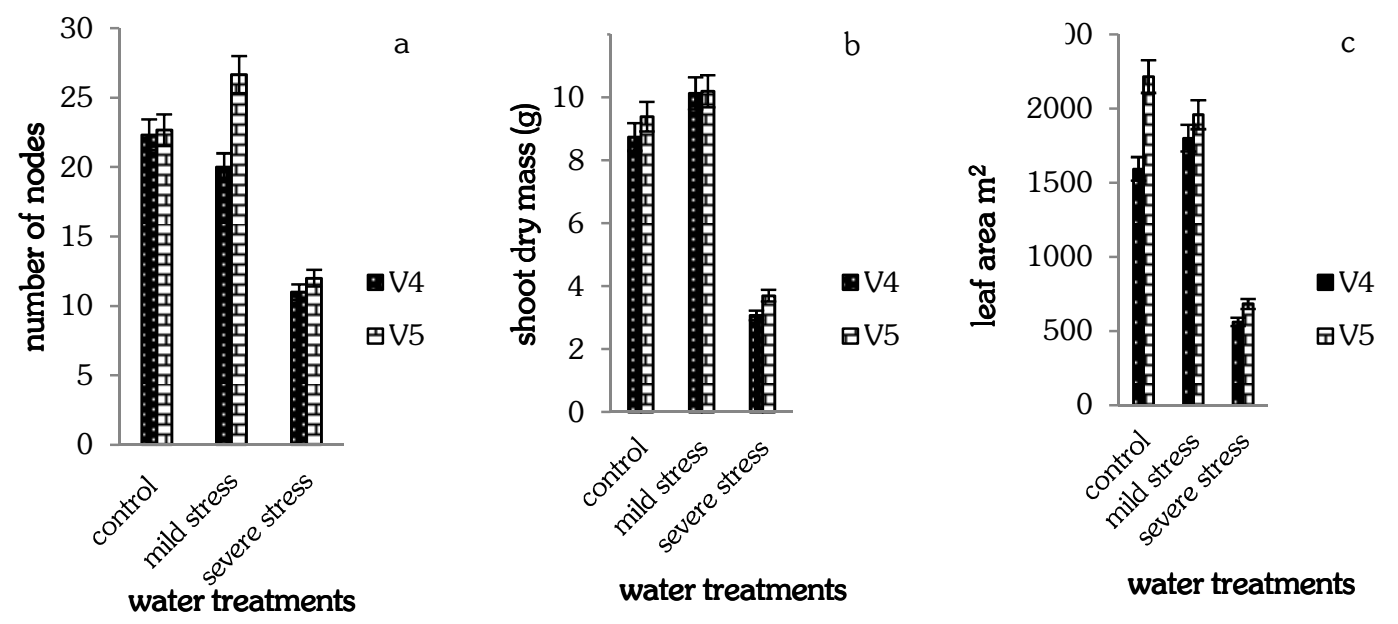

Fig. 2. Number of nodes (a), shoot dry mass (b), and leaf area (c) of soybean grown under well watered condition, mild water stress, and severe water stress. 

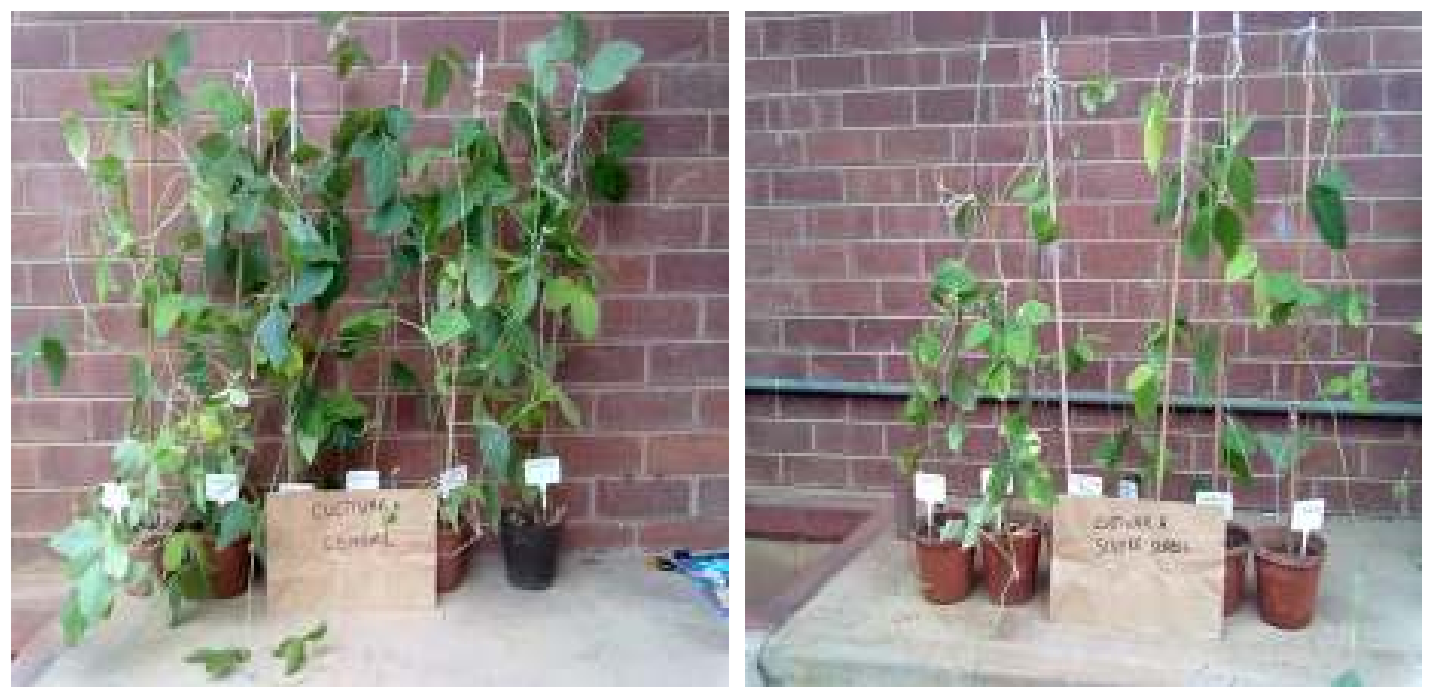

Fig. 3. Effect of water deficit imposed at the vegetative stages $\left(V_{4}\right.$ and $\left.V_{5}\right)$ of soybean. Left $=$ controls, Right $=$ severe stress to $-2.0 \mathrm{MPa}$.
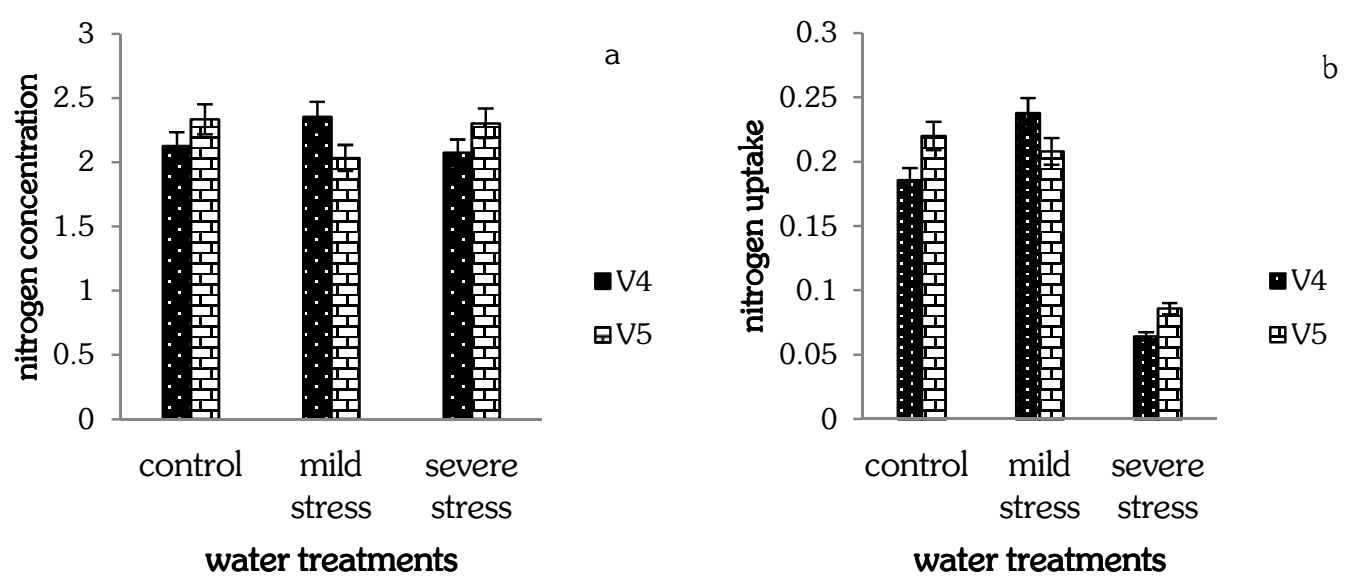

Fig. 4. Effect of water deficits on nitrogen concentration (a) and nitrogen uptake (b) of soybean grown under controlled environment conditions. Each value is derived from mean $( \pm \mathrm{SE})$ of six plants.

The results suggest that severe stress led to significant decrease in nodule number and dry mass (Fig. 1) and is consistent with those of Serraj and Sinclair (1998) with soybean under field and greenhouse conditions. Rhizobium - legume symbiosis establishment is reportedly very sensitive to water deficit (Serraj et al., 1999). The relatively small difference between control and mild stress (Figure 1a and 1b) for nodule number and dry mass is due to the ability of the nodule formation to recover quickly from moderate water stress. Fellows et al. (1987) reported that nodules of soybean were able to recover from water stress of -1.7 MP within 12 hours of rewatering. 
The response of the above ground parameters (Fig. 2) are consistent with those reported from previous studies (Sionit and Kramer 1977). The decrease in leaf area from the severe water stress plants (Fig. 2d) was probably a major cause of decreasing nodule number (Fig. 1a), nodule mass (Fig. 1b) and possibly $\mathrm{N}_{2}$ fixation. A decrease in leaf area is associated with a decrease in the photosynthetic capacity of the plant and hence supply of assimilated to the nodule bacteria. Serraj et al. (1999) reported that a decline in soybean $\mathrm{N}_{2}$ fixation under drought stress is associated with decreased photosynthesis. The well watered plants with heavily branched growth habit contributed to the high above ground parameters like leaf area, shoot dry mass and number of nodes. The plants that were subjected to water stress at both stage $V_{4}$ and $V_{5}$ were not well branched. At stage $V_{4}$ severe stress lowered the number of nodes more than in $V_{5}$ (Fig. 2a). Kadhem et al.(1985) reported that water stress affected node number per plant and pods per node but also found that the effect varied among cultivars which interacted with timing of drought stress.

The nitrogen concentration data ranged between 2 and $2.5 \%$ amongst water treatments. However, the $\mathrm{N}$ content values were lowest for the severe stress and highest for the controls suggesting that those plants that were water stressed severely did not fix enough nitrogen for biomass production compared to the non-stressed ones (Fig. 4).

\section{Conclusion}

At the early vegetative phase nodulation is tolerant to mild stress and subsequent nitrogen fixation can resume at a normal rate. However, if soybean is severely stressed the process of nodulation and nitrogen fixation is reduced irrespective of the vegetative stage at which it is imposed. This has a negative effect on nitrogen content and hence potential for increased productivity. Severe water deficits during the vegetative stage can, therefore, be as important as those in the reproductive phase in terms of their potential for impacting on the productivity of soybean.

\section{References}

Atti, S., R. Bonnell, D. Smith and S. Prasher. 2004. Response of indeterminate soybean (Glycine $\max (\mathrm{L})$ Merr.) to chronic water deficits during reproductive development under greenhouse conditions. Can. Water Res. J. 29: 209-222.

Candogan, B. N. and S. Yazgan. 2016. Yield and quality response of soybean to full and deficit irrigation at different stages of growth under sub-humid climatic conditions. J. Agric. Sci. 22: 129-144.

Castell, S. 2010. Soybean physiology: How well do you know your soybean. www.soybeanstation.org.

Desclaux, D., T. Huynh and P. Roumet. 2000. Identification of soybean plant characteristics that indicate the timing of drought stress. Crop Sci. 40: 716-722.

Fellows, R. J., R. P. Patterson, C. D. Raper Jr. and D. Harris. 1987. Nodule activity and allocation of photosynthates of soybean during recovery from water stress. Plant Physiol. 84: 456460.

Herridge, D. F., M. B. Peoples and R. M. Boddey. 2008. Global input of biological nitrogen fixation in agricultural systems. Plant Soil. 311: 1-18. 
Khadem, F. A., J. E. Specht and J. H. Williams. 1985. Soybean irrigation serially timed from R1 to R6. I. Agronomic responses. Agron. J. 77: 291-298.

Serraj, R. and T. R. Sinclair. 1998. Soybean cultivar variability for nodule formation and growth under drought. Plant Soil. 22: 159-166.

Serraj, R., T. R., Sinclair and L. C. Purcell. 1999. Symbiotic nitrogen fixation response to drought. J. Exp. Bot. 50: 143-155.

Sionit, N. and P. J. Kramer. 1977. Soybean genotypic differences in sensitivity of symbiotic nitrogen fixation to soil dehydration. Plant Soil. 133: 31-37.

Staton, M. 2011. Evaluating soybean nodulation. Michigan State University Extension. Msue.anr.msu.edu/news/evaluating_soybean_nodulation. Downloaded 17 August 2017. 